\title{
THE MODIFIED COLLOCATION TREFFTZ METHOD AND LAPLACIAN DECOMPOSITION FOR SOLVING TWO-DIMENSIONAL STOKES PROBLEMS
}

\author{
Chia-Ming Fan \\ Department of Harbor and River Engineering \& Computation and Simulation Center, National Taiwan Ocean University, \\ Keelung 20224, Taiwan, R.O.C., cmfan@ntou.edu.tw \\ Hong-Huei Li \\ Department of Harbor and River Engineering \& Computation and Simulation Center, National Taiwan Ocean University, \\ Keelung 20224, Taiwan, R.O.C. \\ Chung-Lun Kuo \\ Department of Systems Engineering and Naval Architecture, National Taiwan Ocean University, Keelung 20224, \\ Taiwan, R.O.C.
}

Follow this and additional works at: https://jmstt.ntou.edu.tw/journal

Part of the Engineering Commons

\section{Recommended Citation}

Fan, Chia-Ming; Li, Hong-Huei; and Kuo, Chung-Lun (2011) "THE MODIFIED COLLOCATION TREFFTZ METHOD AND LAPLACIAN DECOMPOSITION FOR SOLVING TWO-DIMENSIONAL STOKES PROBLEMS," Journal of Marine Science and Technology. Vol. 19: Iss. 5, Article 8.

DOI: $10.51400 / 2709-6998.2166$

Available at: https://jmstt.ntou.edu.tw/journal/vol19/iss5/8

This Research Article is brought to you for free and open access by Journal of Marine Science and Technology. It has been accepted for inclusion in Journal of Marine Science and Technology by an authorized editor of Journal of Marine Science and Technology. 


\title{
THE MODIFIED COLLOCATION TREFFTZ METHOD AND LAPLACIAN DECOMPOSITION FOR SOLVING TWO-DIMENSIONAL STOKES PROBLEMS
}

\author{
Chia-Ming Fan*, Hong-Huei Li*, and Chung-Lun Kuo**
}

Key words: modified collocation Trefftz method, Laplacian decomposition, Stokes problem, boundary-type meshless method, T-complete functions.

\begin{abstract}
In this paper, the two-dimensional Stokes problem is analyzed by the modified collocation Trefftz method (MCTM) and the Laplacian decomposition. The coupled Stokes equations are converted to three Laplace equations by utilizing the Laplacian decomposition and then the boundary-type meshless MCTM is adopted to solve the resultant Laplace equations. The MCTM, free from mesh and numerical quadrature, is derived from the conventional Trefftz method by considering the characteristic length of the domain, which stabilize the numerical scheme and obtain highly accurate results. Besides, the solutions are expressed as the linear combination of T-complete functions and the velocity as well as pressure are coupled by collocating the boundary conditions. Several numerical examples are provided to demonstrate the efficacy and accuracy of the proposed meshless scheme. In addition, the numerical results demonstrates that the proposed meshless scheme can solve the Stokes problems accurately in simplyand doubly-connected domains.
\end{abstract}

\section{INTRODUCTION}

Due to the complexity of the governing equations for viscous incompressible flow, it is almost impossible to mathematically obtain the closed-form solutions of the Navier-Stokes equations without any simplification. Under the assumption of negligible inertia force, the Navier-Stokes equations can be reduced to the Stokes equations, which are used to describe

Paper submitted 03/18/10; revised 05/04/10; accepted 06/05/10. Author for correspondence: Chia-Ming Fan (e-mail: cmfan@ntou.edu.tw).

*Department of Harbor and River Engineering \& Computation and Simulation Center, National Taiwan Ocean University, Keelung 20224, Taiwan, R.O.C.

**Department of Systems Engineering and Naval Architecture, National Taiwan Ocean University, Keelung 20224, Taiwan, R.O.C. very slow flow field or motion of extremely viscous fluid. Although the governing equations can be simplified, it is still very difficult to obtain the solutions of Stokes equations by mathematical ways. Owing to the quick developments of computer technology in the past decades, the numerical simulation seems to be the better choice for understanding the fluid flow.

When numerical simulation is considered for flow field, the governing equations can be classified as: the primitive-variables formulation $[14,19]$, the velocity-vorticity formulation $[5,17]$, the stream function-vorticity formulation and the stream function formulation [16]. Among them, only the primitive-variables formulation can directly acquire the distribution of pressure. Besides, systems of inhomogeneous partial differential equations, which can not be directly resolved by boundary-type numerical methods, will appear if the velocity-vorticity formulation and the stream function-vorticity formulation are considered. Therefore, it is very important to develop an efficient numerical scheme for solving the primitive-variables formulation of the Stokes equations. Because the primitive-variables formulation is a system of coupled equations, it is not easy to analyze the Stokes equations directly. In 2007, Curteanu et al. [4] used the Laplacian decomposition to convert the Stokes equations to three Laplace equations which can greatly simplify the governing equations for numerical schemes. Hence, in this paper, the Laplacian decomposition is adopted to transform the governing equations for the Stokes flow.

There are some numerical scheme can be used for analyzing the Stokes equations, such as the finite difference method (FDM) [14], finite element method (FEM), boundary element method (BEM) $[5,20]$, etc. The FDM, FEM and BEM belongs to the mesh-dependent numerical methods. Hence, the time-consuming mesh generation and the troublesome numerical quadrature are needed during numerical implementation. In contract to those mesh-dependent methods, there are some numerical schemes which are classified as the meshindependent (meshless) methods. The most well-known meshless methods are the multiquadric (MQ) method [18], the boundary particle method [2], the method of fundamental 
solutions (MFS) $[6,16,17,19]$, the Trefftz method [3, 8, 1013], etc. Young et al. [18] adopted the MQ method for solving the velocity-vorticity formulation of the Stokes equation, while Young et al. [19] used the MFS and the Stokeslet to analyze the primitive-variable formulation of the Stokes equations.

Among those meshless methods, the MFS and the Trefftz method are the most famous methods since both of them are the boundary-type meshless methods. The solution of MFS is expressed as the linear combination of fundamental solutions which are located out of the computational domain $[6,16$, 17, 19]. The positions of the fundamental solutions will have great influence on the accuracy of the numerical results. Though many researchers proposed practical ways to locate the source, it will cost extra numerical burdens in implementation. On the other hand, the solution of Trefftz method is expressed as the linear combination of T-complete functions [8]. The unknown coefficients can be found by collocating the boundary conditions. Kita and Kamiya [8] classified different kind of Trefftz methods and published a review for the Trefftz methods. Recently, Li et al. [9] compared the Trefftz methods with other boundary methods. Among the previous studies, it is found that the condition number of the matrix in Trefftz method is extremely high. Therefore, the numerical solutions will become very unstable and inaccurate when more nodes are used.

Because of the ill-conditioning matrix, the engineering applications of the Trefftz method are seldom. In 2007, Liu $[12,13]$ proposed the modified collocation Trefftz method (MCTM) by introducing the characteristic length of the computational domain. From his results, it is proved that the condition number of the matrix can be greatly reduced. Hence the solution of the MCTM becomes very stable and extremely accurate. Furthermore, Liu [10] extended the MCTM to doubly-connected domain by considering two different characteristic lengths, while Chen et al. [3] demonstrated the ability of the MCTM for dealing with the problem in high-aspect ratio domain. Hence, the MCTM retains the advantages of the conventional Trefftz method and, in the meantime, gets rid of the ill-conditioning matrix. So, the meshless MCTM will be adopted in this study for analyzing the Laplace equations, which are converted from the Stokes equations by Laplacian decomposition.

In this paper, the primitive-variable formulation of the Stokes equations will be converted to three Laplace equations by the Laplacian decomposition. Then, the solutions of the Laplace equations are expressed as the linear combination of T-complete functions according to the MCTM, which is meshless and integral-free. The unknown coefficients can be obtained by directly collocating the boundary conditions. Once the coefficients are obtained, the numerical solutions and their derivatives at any position inside computational domain can be acquired by simple summation. Unlike other formulations for the Stokes equations, the distribution of pressure through the computational domain can be obtained directly. The Stokes equations and the Laplacian decomposition are described after the brief introduction of the motivation for this study. Then, four numerical examples are provided to show the efficiency and accuracy of the proposed numerical scheme. Finally, some conclusions are drawn according to the numerical performance.

\section{MATHEMATICAL FORMULATIONS}

\section{Stokes Equations}

The governing equation of the viscous incompressible flow is the Navier-Stokes equations. When the viscous force is very large in comparing with the inertial force, the equations can be reduced to the Stokes equations,

$$
\begin{aligned}
& \frac{\partial u}{\partial x}+\frac{\partial v}{\partial y}=0, \quad(x, y) \in \Omega, \\
& \Delta u-\frac{\partial p}{\partial x}=0, \quad(x, y) \in \Omega, \\
& \Delta v-\frac{\partial p}{\partial y}=0, \quad(x, y) \in \Omega,
\end{aligned}
$$

where, $u(x, y)$ and $v(x, y)$ are $x$-directional and $y$-directional velocity components. $p(x, y)$ is the pressure and $\Delta$ and the Laplacian operator. $\Omega$ denotes the computational domain. The above system of equations can be solved with suitable boundary conditions.

Eq. (1) is the continuity equation. Eqs. (2) and (3) are the $x$-directional and $y$-directional momentum equations, respectively. By observing the system of equations, the velocity components and pressure are coupled in these equations. So, it is not easy to analyze this system of equations directly. Since the unknowns in Eqs. (1)-(3) are velocity and pressure of the flow field, these three equations form the well-known primitivevariable formulation of the Stokes equations.

\section{Laplacian Decomposition}

Differentiating of Eq. (2) with respect to $x$ and differentiating of Eq. (3) with respect to $y$ forms the following equation,

$$
\Delta p=0 .
$$

In order to simplify Eqs. (1)-(3), the following unknown variables are introduced,

$$
\begin{aligned}
& f(x, y)=u(x, y)-\frac{x}{2} p(x, y), \\
& g(x, y)=v(x, y)-\frac{y}{2} p(x, y) .
\end{aligned}
$$


By taking Laplacian to Eqs. (5) and (6), the following equations can be acquired,

$$
\begin{aligned}
& \Delta f=\Delta\left(u-\frac{x}{2} p\right)=\Delta u-\frac{\partial p}{\partial x}=0, \\
& \Delta g=\Delta\left(v-\frac{y}{2} p\right)=\Delta v-\frac{\partial p}{\partial y}=0,
\end{aligned}
$$

where Eqs. (7) and (8) are equivalent to the momentum equations of the Stokes equations, Eqs. (2) and (3).

Hence, by using the Laplacian decomposition, the Stokes equations, Eqs. (1)-(3), are converted to three Laplace equations, Eqs. (4), (7) and (8). The transformation can reduce the difficulties in solving the governing equations of the Stokes flow.

\section{Using the Continuity Equation as the Augmented Boundary Condition}

From the descriptions in previous subsection, the Stokes equations can be transformed to three Laplace equations. If we follow the mathematical derivation conversely, we should find that the continuity equation is not satisfied inside computational domain. From Eq. (4), the following equation can be derived,

$$
\Delta\left(\frac{\partial u}{\partial x}+\frac{\partial v}{\partial y}\right)=0 \quad(x, y) \in \Omega
$$

It can be easily found that Eq. (1) is not equal to Eq. (9). From Eq. (4), only Eq. (9) can be derived, not Eq. (1). Fortunately, according to the maximum-minimum theorem of the Laplace equation [7], Eq. (9) with the following boundary condition is equivalent to Eq. (1),

$$
\frac{\partial u}{\partial x}+\frac{\partial v}{\partial y}=0, \quad(x, y) \in \Gamma
$$

where, $\Gamma$ denotes the boundary along the computational domain, $\Omega$.

Consequently, the Stokes equations, Eqs. (1)-(3), is equivalent to three Laplace equations, Eqs. (4), (7), (8), with the continuity equation as the augmented boundary condition, Eq. (10). The readers, who interest in more rigorous descriptions of the transformation, can find the details in [4].

\section{MODIFIED COLLOCATION TREFFTZ METHOD (MCTM)}

In this section, the MCTM for Laplace equation is introduced. When the following potential problem is considered,

$$
\Delta w=0, \quad(x, y) \in \Omega
$$

where the corresponding boundary of the domain in the polar coordinates is given by $\Gamma=\{(r, \theta) \mid r=\rho(\theta), 0 \leq \theta \leq 2 \pi\}$.

It is known that the T-complete functions [8] for two-dimensional Laplace equation can be shown as:

$$
\left\{1, r^{k} \cos (k \theta), r^{k} \sin (k \theta), k=1,2,3, \ldots \ldots \ldots\right\} .
$$

The numerical solution can be expanded in terms of the above bases which forms the conventional Trefftz method. Instead of the above bases, Liu [10-13] suggested to modify the T-complete functions by considering the characteristic length of the computational domain,

$$
\left\{1,\left(\frac{r}{R_{0}}\right)^{k} \cos (k \theta),\left(\frac{r}{R_{0}}\right)^{k} \sin (k \theta), k=1,2,3, \ldots \ldots . .\right\},
$$

where $R_{0} \geq \rho_{\max }=\max _{\theta \in[0,2 \pi]} \rho(\theta) . R_{0}$ is the characteristic length of the computational domain. In MCTM, the solutions are expressed by the modified T-complete functions, Eq. (13), instead of the original bases, Eq. (12). Once $R_{0}$ is equal to one, the formulation of MCTM becomes the conventional Trefftz method.

In MCTM, the numerical solution of the Laplace equation, Eq. (11), can be expressed as the linear combination of modified T-complete functions, Eq. (13),

$$
\begin{aligned}
w(x, y) & =a_{0}^{w}+\sum_{k=1}^{\infty} a_{k}^{w}\left(\frac{r}{R_{0}}\right)^{k} \cos (k \theta)+b_{k}^{w}\left(\frac{r}{R_{0}}\right)^{k} \sin (k \theta) \\
& \cong a_{0}^{w}+\sum_{k=1}^{\infty} a_{k}^{w} \phi_{k}+b_{k}^{w} \psi_{k},
\end{aligned}
$$

where $a_{0}^{w}$, and $\left\{a_{k}^{w}\right\}_{k=1}^{m}$ and $\left\{b_{k}^{w}\right\}_{k=1}^{m}$ are the unknown coefficients which can be found by collocating the boundary conditions. In Eq. (14), we used $m$ terms to truncate the infinite series in the original expressions due to the limitation of numerical simulation. The choice of $m$ will be determined by the boundary condition and the smoothness of the solution, so we may expect to use larger $m$ to avoid any possible error due to the insufficient terms in the solution expression. When the coefficients are determined by collocating the boundary conditions, the numerical solution and its derivative at any position inside the computational domain can be found by simple summation, Eq. (14). In Liu's studies [10-13], the condition number of the coefficient matrix can be greatly reduced. Therefore, the MCTM is very stable with respect to noise and the numerical results are highly accurate.

Following the similar procedure, the solutions of Eqs. (4), (7), (8) can be expressed as: 


$$
\begin{aligned}
& p(x, y)=a_{0}^{p}+\sum_{k=1}^{m} a_{k}^{p} \phi_{k}+b_{k}^{p} \psi_{k}, \\
& f(x, y)=a_{0}^{f}+\sum_{k=1}^{m} a_{k}^{f} \phi_{k}+b_{k}^{f} \psi_{k}, \\
& g(x, y)=a_{0}^{g}+\sum_{k=1}^{m} a_{k}^{g} \phi_{k}+b_{k}^{g} \psi_{k},
\end{aligned}
$$

where $a_{0}^{p},\left\{a_{k}^{p}\right\}_{k=1}^{m},\left\{b_{k}^{p}\right\}_{k=1}^{m}, a_{0}^{f},\left\{a_{k}^{f}\right\}_{k=1}^{m},\left\{b_{k}^{f}\right\}_{k=1}^{m}, a_{0}^{g},\left\{a_{k}^{g}\right\}_{k=1}^{m}$ and $\left\{b_{k}^{g}\right\}_{k=1}^{m}$ are the unknown coefficients. Since $p, f$ and $g$ can be expressed as the combination of T-complete functions, we can find the expressions of velocity components via Eqs. (5)(6),

$$
\begin{aligned}
& u(x, y)=a_{0}^{f}+\sum_{k=1}^{m} a_{k}^{f} \phi_{k}+b_{k}^{f} \psi_{k}+\frac{x}{2}\left(a_{0}^{g}+\sum_{k=1}^{m} a_{k}^{g} \phi_{k}+b_{k}^{g} \psi_{k}\right) \\
& v(x, y)=a_{0}^{g}+\sum_{k=1}^{m} a_{k}^{g} \phi_{k}+b_{k}^{g} \psi_{k}+\frac{y}{2}\left(a_{0}^{g}+\sum_{k=1}^{m} a_{k}^{g} \phi_{k}+b_{k}^{g} \psi_{k}\right)
\end{aligned}
$$

Since the velocity components and pressure can be expressed as summations of T-complete functions, the unknown coefficients can be determined by collocating the physicsbased boundary conditions and the continuity equation, Eq. (10). From previous descriptions of the numerical procedure, it can be found that the MCTM is free from mesh and numerical integration. In addition, the derivatives of velocity components and pressure can be derived by differentiating Eqs. (15), (18), (19) directly. Utilizing the Laplacian decomposition, the Stokes equations can be converted to three Laplace equations which can be solved by MCTM. The efficiency of the proposed meshless scheme can be validated through several numerical examples in the following sections.

\section{NUMERICAL RESULTS AND COMPARISONS}

In order to validate the efficacy and demonstrate the accuracy of the proposed numerical scheme, four examples are adopted. Three of them are simply-connected domains and the other is the doubly-connected domain.

\section{Example 1: Lid-driven Cavity Flow}

The first example is the well-known lid-driven cavity. The schematic diagram of the cavity flow is shown in Fig. 1. The lid is moving with unit velocity in $x$ direction and the boundary conditions in the other sides are assumed as no-slip.

Except for the velocity boundary conditions, the continuity equation should be forced along the boundary, Eq. (10), which is explained previously. The reference point for pressure $(p=1)$ is given at $(x, y)=(1,0)$ to uniquely determine the

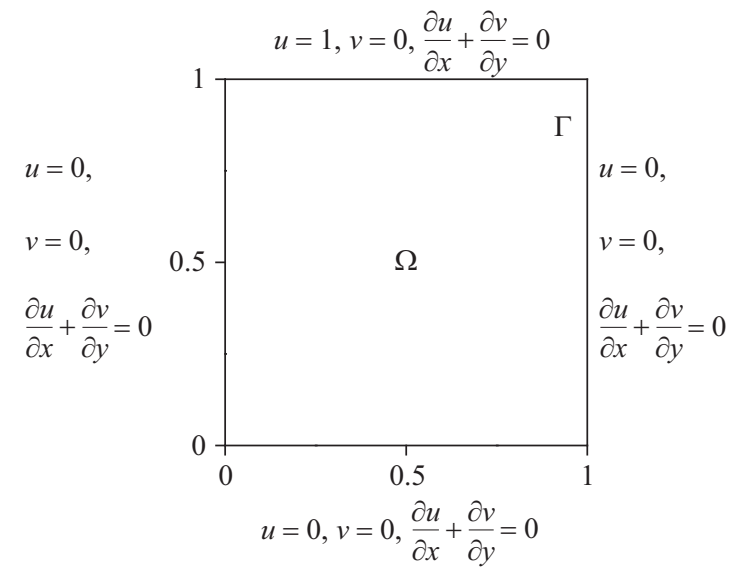

Fig. 1. The schematic diagram of computational domain and boundary condition for example 1.

distribution of pressure. By observing the momentum equations, its physical meaning is the balance between the pressure force and viscous force. The pressure force is interpreted by the pressure gradient term. Therefore, the derivative of pressure is very important in comparing with the distribution of pressure. That means if we add a constant to the pressure distribution, the modified distribution of pressure still satisfies the governing equations. So, in numerical simulation, we will impose a reference pressure which denotes the measureable pressure in realistic applications.

In this numerical experiment, 61 boundary nodes are used and $m$ is set as 30 . The profiles of velocity along $x=0.5$ and $y=0.5$ are depicted in Figs. 2(a) and 2(b), respectively. The numerical results are almost identical with MFS solution [19]. Besides, the distribution of velocity vector is shown in Fig. 3. In Fig. 3, it can be found that the circulation is formed by the influence of the moving lid. It should be emphasized that the velocity components at any position can be calculated once the coefficients are obtained. The density of vector in Fig. 3 is used for demonstration only. If more details of flow field are required, it will not cost a lot from the original procedure.

\section{Example 2}

In the second example, the following analytical solutions are used [4]:

$$
\begin{gathered}
u(x, y)=2 x y+\frac{y^{3}}{6}, \\
v(x, y)=x^{2}-y^{2}+\frac{x^{3}}{6}, \\
p(x, y)=x y,
\end{gathered}
$$

where the square computational domain is used and the velocity components, which are derived directly from the analytical solutions, are specified along the boundary. In this test, 


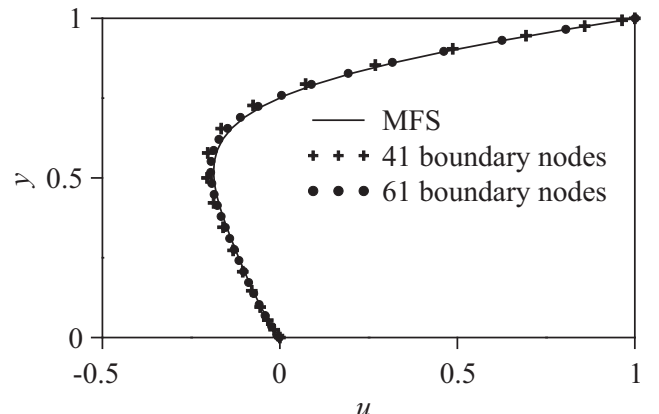

(a)

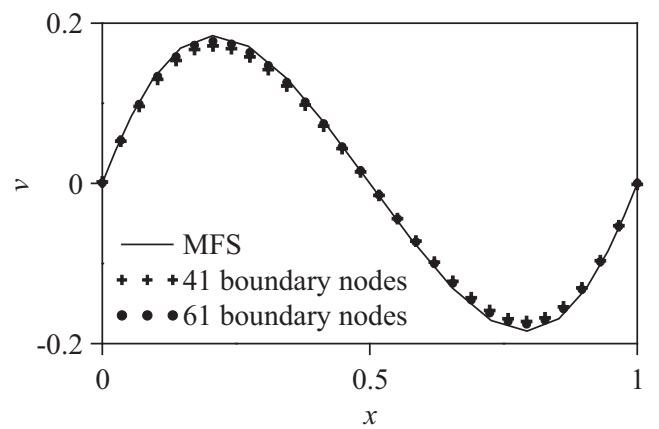

(b)

Fig. 2. The profile of (a) $u$ at $x=0.5$ along $y$ axis and (b) $v$ at $y=0.5$ along $x$ axis.

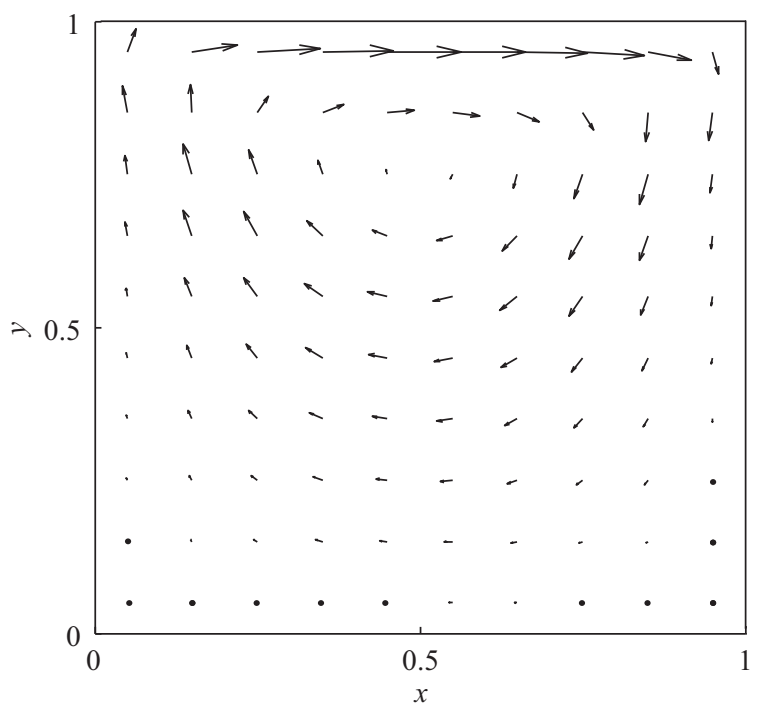

Fig. 3. The distribution of velocity vector for example 1.

101 boundary nodes is adopted and $m$ is set as 50 . In addition, the reference point for pressure, which can be derived from the analytical solution, is given at $(x, y)=(0.5,-0.5)$. In this test, the maximum radius of physical domain, $\rho_{\max }$ is $0.5 \sqrt{2}$, so the characteristic length that we adopted is one. In Fig. 4, the contours of $u, v$ and $p$ are demonstrated. Since the results are almost identical to the analytical solutions, only the numerical results are present. In Fig. 5, the distributions of absolute error of these three components are shown. The error for $u$ and $v$

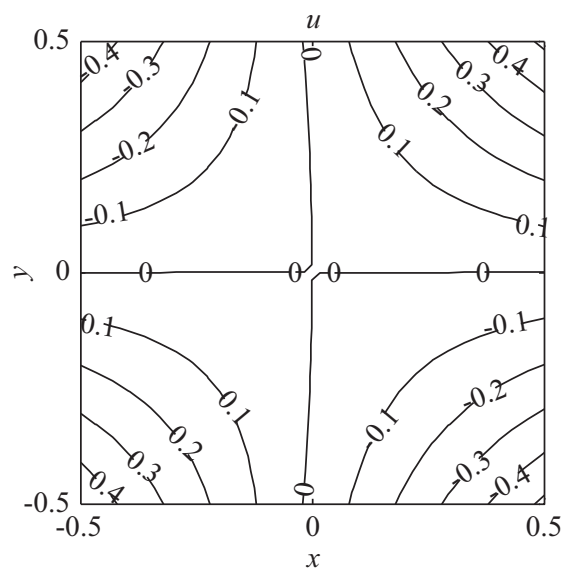

(a) $x$-directional velocity

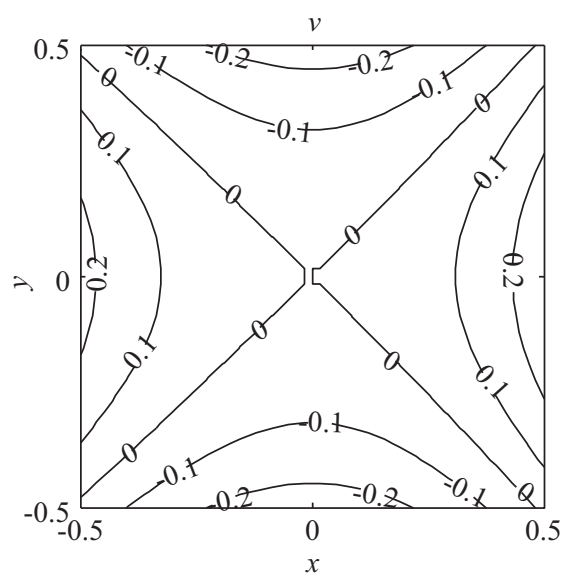

(b) $y$-directional velocity

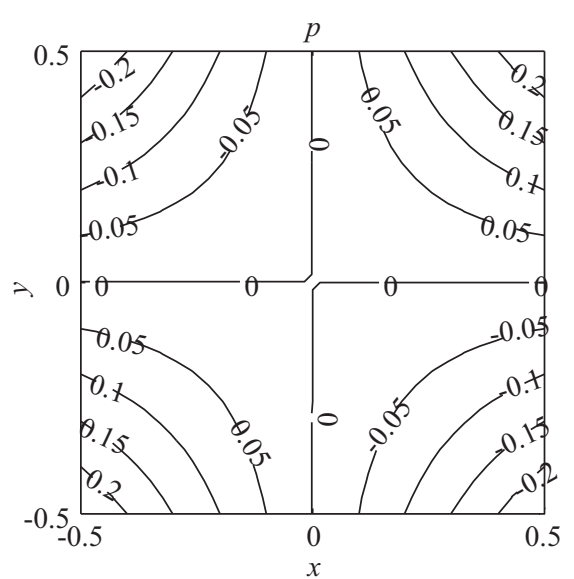

(c) pressure

Fig. 4. The distributions of (a) $x$-directional velocity, (b) $y$-directional velocity and (c) pressure.

are about $10^{-11}$ as well as the error for $p$ is about $10^{-8}$. From the comparisons, it can be found that the numerical results are highly accurate due to the use of characteristic length. In order to compare the results by the proposed approach with solutions by the conventional Trefftz method, the numerical comparisons by using different characteristic lengths and different 
Table 1. The comparisons of Maximum Absolute Error (MAE) in example 2 by adopting different origins for Trefftz bases. $(\varepsilon=0.5)$

(a) Origin for Trefftz bases at $(0,0)$

\begin{tabular}{|c|c|c|c|c|}
\hline MAE & $R_{0}=1$ & $R_{0}=0.5 \sqrt{2}+\varepsilon$ & $R_{0}=2$ & $R_{0}=3$ \\
\hline$u$ & $2.19 \mathrm{e}-011$ & $1.16 \mathrm{e}-011$ & $3.37 \mathrm{e}-011$ & $2.55 \mathrm{e}-010$ \\
\hline$v$ & $1.93 \mathrm{e}-011$ & $1.42 \mathrm{e}-011$ & $3.47 \mathrm{e}-011$ & $2.57 \mathrm{e}-010$ \\
\hline$p$ & $8.03 \mathrm{e}-009$ & $3.41 \mathrm{e}-009$ & $2.37 \mathrm{e}-009$ & $2.60 \mathrm{e}-008$ \\
\hline
\end{tabular}

(b) Origin for Trefftz bases at $(-0.2,-0.2)$

\begin{tabular}{|c|c|c|c|c|}
\hline MAE & $R_{0}=1$ & $R_{0}=\sqrt{(0.5-(-0.2))^{2}+(0.5-(-0.2))^{2}}+\varepsilon$ & $R_{0}=2$ & $R_{0}=3$ \\
\hline$u$ & $5.32 \mathrm{e}-007$ & $1.11 \mathrm{e}-010$ & $2.28 \mathrm{e}-011$ & $3.17 \mathrm{e}-011$ \\
\hline$v$ & $9.81 \mathrm{e}-007$ & $1.19 \mathrm{e}-010$ & $2.29 \mathrm{e}-011$ & $3.61 \mathrm{e}-011$ \\
\hline$p$ & $3.23 \mathrm{e}-004$ & $1.30 \mathrm{e}-008$ & $2.60 \mathrm{r}-009$ & $1.41 \mathrm{e}-009$ \\
\hline
\end{tabular}

(c) Origin for Trefftz bases at $(-0.3,-0.3)$

\begin{tabular}{|c|c|c|c|c|}
\hline MAE & $R_{0}=1$ & $R_{0}=\sqrt{(0.5-(-0.3))^{2}+(0.5-(-0.3))^{2}}+\varepsilon$ & $R_{0}=2$ & $R_{0}=3$ \\
\hline$u$ & 0.2195 & $4.85 \mathrm{e}-009$ & $7.14 \mathrm{e}-010$ & $2.66 \mathrm{e}-010$ \\
\hline$v$ & 0.1174 & $3.79 \mathrm{e}-009$ & $7.12 \mathrm{e}-010$ & $2.75 \mathrm{e}-010$ \\
\hline$p$ & 34.541 & $5.29 \mathrm{e}-007$ & $8.34 \mathrm{e}-008$ & $1.82 \mathrm{e}-008$ \\
\hline
\end{tabular}

(d) Origin for Trefftz bases at $(-0.4,-0.4)$

\begin{tabular}{|c|c|c|c|c|}
\hline MAE & $R_{0}=1$ & $R_{0}=\sqrt{(0.5-(-0.4))^{2}+(0.5-(-0.4))^{2}}+\varepsilon$ & $R_{0}=2$ & $R_{0}=3$ \\
\hline$u$ & 0.6082 & $3.34 \mathrm{e}-008$ & $7.97 \mathrm{e}-009$ & $3.95 \mathrm{e}-010$ \\
\hline$v$ & 0.4671 & $3.07 \mathrm{e}-008$ & $8.42 \mathrm{e}-009$ & $3.51 \mathrm{e}-010$ \\
\hline$p$ & 129.54 & $3.68 \mathrm{e}-006$ & $1.16 \mathrm{e}-006$ & $4.41 \mathrm{e}-008$ \\
\hline
\end{tabular}

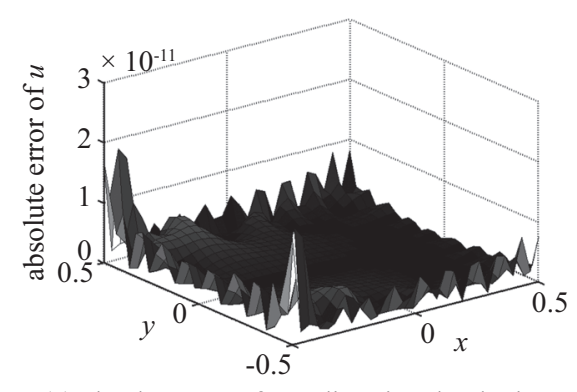

(a) absolute error for $x$-directional velocity

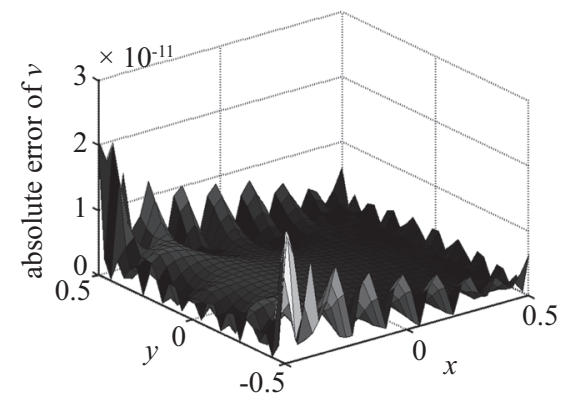

(b) absolute error for $y$-directional velocity

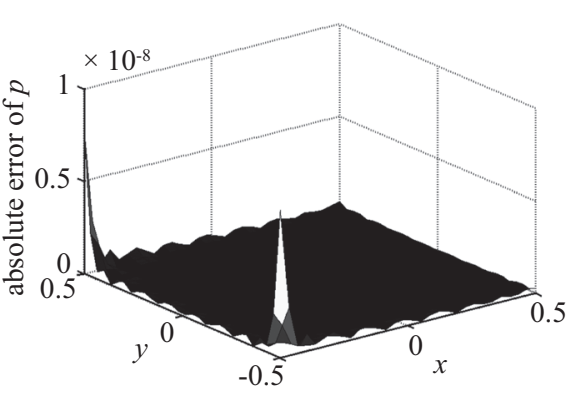

(c) absolute error for pressure

Fig. 5. The distributions of absolute error for (a) $x$-directional velocity, (b) $y$-directional velocity and (c) pressure.

origins for Trefftz bases are shown in Tables 1(a)-(d).

In these tables, four different characteristic lengths are used. When the characteristic length is equal to one, the MCTM is identical to the conventional Trefftz method. From the comparisons of maximum absolute errors for velocity components and pressure, it can be found that the results by conventional approach are very sensitive to the origins of the Trefftz bases. In contrary, the errors by MCTM are very stable with respect to different origin locations.

\section{Example 3: General Flow in a Channel}

After the validations from the previous two examples, the third example considers more practical case [4]. The schematic diagram of the test is depicted in Fig. 6.

The uniform inflow velocity is assumed at the left boundary. The upper and bottom boundary conditions are considered as no-slip. In addition, the parabolic profile of outflow velocity is used in the right boundary. In this test, 301 boundary nodes is used and $m$ is set as 150 . The distribution of $\mathrm{x}$-directional velocity is demonstrated in Fig. 7 when different characteristic lengths are used. In Fig. 7(a), the characteristic length is set as one in which the MCTM is equivalent to the convectional Trefftz method. Due to the ill-conditioning matrix, the solutions in Fig. 7(a) is asymmetric with respect to $y=0$. On the 


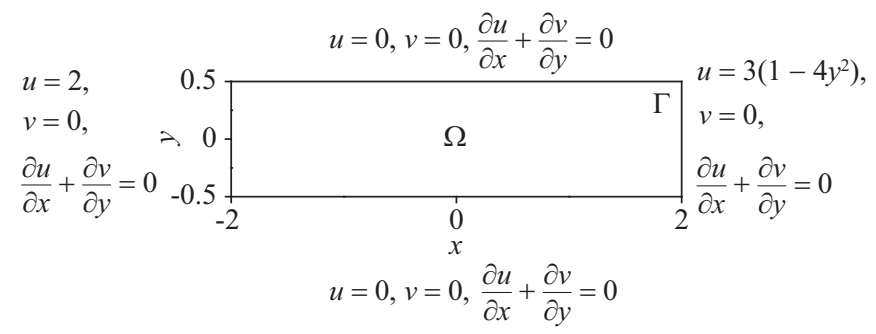

Fig. 6. The schematic diagram of computational domain and boundary condition for example 3 .

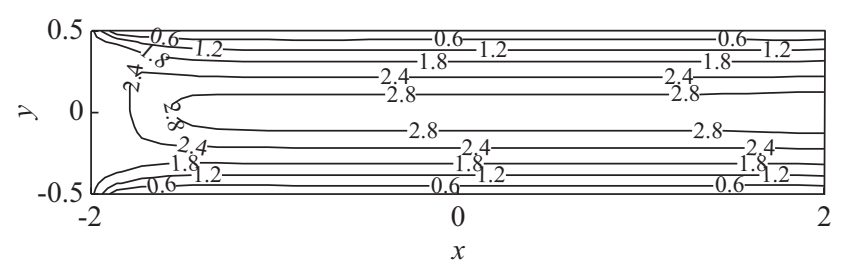

(a) $R_{0}=1$

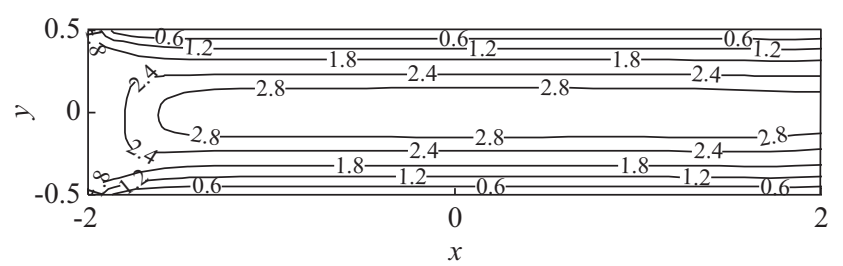

(a) $R_{0}=\sqrt{4.25}$

Fig. 7. The distributions of $x$-directional velocity for (a) $R_{0}=1$ and (b) $R_{0}=\sqrt{4.25}$.

other hand, the characteristic length in Fig. 7(b) is $\sqrt{4.25}$ Therefore, this distribution in Fig. 7(b) is similar with the published results [4]. Following the successful validation, it is believed that the results are accurate in comparing with the solution by BEM [4]. In addition, it is proved that the use of characteristic length can stabilize the numerical scheme and obtain accurate solutions.

\section{Example 4}

The computational domain of the fourth example is a doubly-connected domain which is shown in Fig. 8. This boundary conditions and the geometry are the same as Young et al. [16]. The left and right boundaries are assumed as non-slip. In addition, the upper and bottom boundaries are moving by constant speed in positive and negative $\mathrm{x}$ directions. Inside the square domain, there is a fixed cylinder. The Stokes flow in such irregular domain is not easily to simulated by any mesh-dependent methods. By using the proposed method, only the boundary nodes are needed which will greatly simplify the numerical methods for Stokes problems.

Since the computational domain is a doubly-connected one, the problem can be considered as linear combination of an exterior problem and an interior problem. Therefore, the solutions of MCTM, Eq. (14), should be changed to the following form,

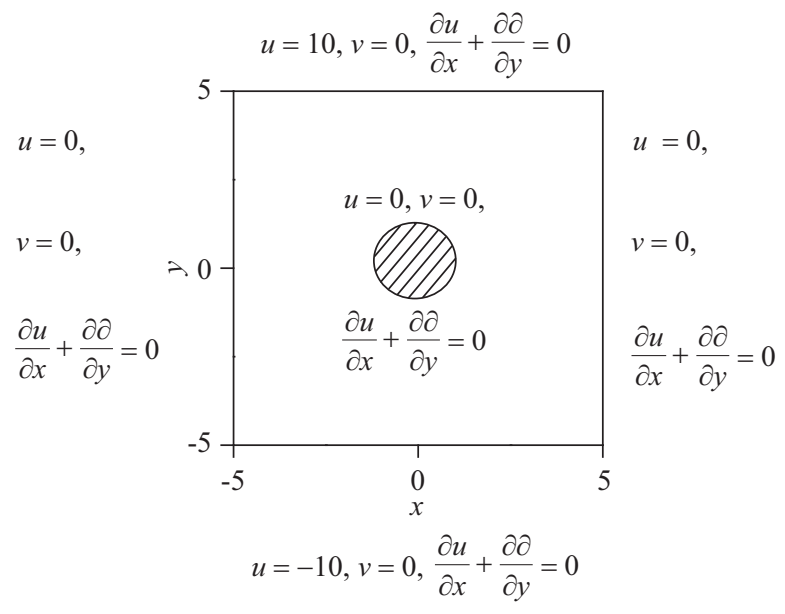

Fig. 8. The schematic diagram of computational domain and boundary condition for example 4.

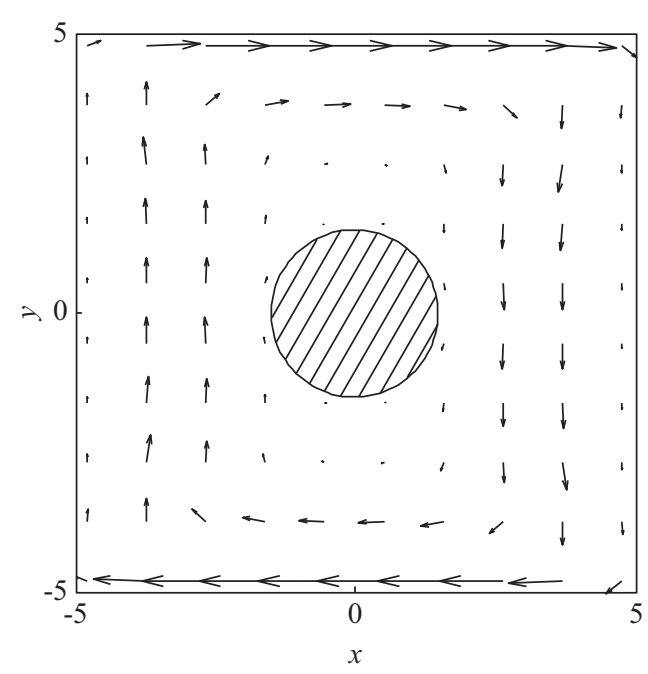

Fig. 9. The distribution of velocity vector for example 4.

$$
\begin{aligned}
w(x, y)=a_{0}^{w} & +\sum_{k=1}^{m} a_{k}^{w}\left(\frac{r}{R_{0}}\right)^{k} \cos (k \theta)+b_{k}^{w}\left(\frac{r}{R_{0}}\right)^{k} \sin (k \theta) \\
& +c_{0}^{w} \ln \left(\frac{r}{R_{1}}\right)+\sum_{k=1}^{m} c_{k}^{w}\left(\frac{r}{R_{1}}\right)^{-k} \cos (k \theta) \\
& +d_{k}^{w}\left(\frac{r}{R_{1}}\right)^{-k} \sin (k \theta)
\end{aligned}
$$

where $c_{0}^{w},\left\{c_{k}^{w}\right\}_{k=1}^{m}$ and $\left\{d_{k}^{w}\right\}_{k=1}^{m}$ are the unknown coefficients corresponding to the exterior problem. The detailed explanation can be referred to Liu [10]. $R_{0}$ and $R_{1}$ are the characteristic lengths for the interior and exterior problems. In the numerical experiment, $R_{0}=5 \sqrt{2}$ and $R_{1}=0.75$ are used.

The Eqs. (15)-(19) should be changed by the similar way. By collocating the velocity boundary conditions and the con- 
Table 2. The numerical solution and comparison of example 4.

\begin{tabular}{|c|c|c|c|c|c|c|c|}
\hline \multicolumn{4}{|c|}{ Channel wall } & \multicolumn{4}{c|}{ Cylinder boundary } \\
\hline$(x, y)$ & Present & $\begin{array}{c}\text { Analytical } \\
\text { solution }\end{array}$ & $\begin{array}{c}\text { Absolute } \\
\text { Error }\end{array}$ & $(x, y)$ & Present & $\begin{array}{c}\text { Analytical } \\
\text { solution }\end{array}$ & $\begin{array}{c}\text { Absolute } \\
\text { Error }\end{array}$ \\
\hline$(-5.00,-4.75)$ & $2.18 \mathrm{E}-13$ & 0.00 & $1 \mathrm{E}-13$ & $(1.50,0.00)$ & $-5.98 \mathrm{E}-14$ & 0.00 & $1 \mathrm{E}-14$ \\
\hline$(-5.00,-2.25)$ & $-1.14 \mathrm{E}-12$ & 0.00 & $1 \mathrm{E}-12$ & $(1.21,0.88)$ & $5.77 \mathrm{E}-14$ & 0.00 & $1 \mathrm{E}-14$ \\
\hline$(-5.00,0.25)$ & $8.50 \mathrm{E}-13$ & 0.00 & $1 \mathrm{E}-13$ & $(0.46,1.43)$ & $1.80 \mathrm{E}-13$ & 0.00 & $1 \mathrm{E}-13$ \\
\hline$(-5.00,2.75)$ & $-2.59 \mathrm{E}-13$ & 0.00 & $1 \mathrm{E}-13$ & $(-0.46,1.43)$ & $7.53 \mathrm{E}-14$ & 0.00 & $1 \mathrm{E}-14$ \\
\hline$(-5.00,4.75)$ & $2.02 \mathrm{E}-13$ & 0.00 & $1 \mathrm{E}-13$ & $(-1.21,0.88)$ & $-6.71 \mathrm{E}-14$ & 0.00 & $1 \mathrm{E}-14$ \\
\hline$(-4.75,5.00)$ & 9.99 & 10.00 & $1 \mathrm{E}-13$ & $(-1.50,0.00)$ & $-1.80 \mathrm{E}-13$ & 0.00 & $1 \mathrm{E}-13$ \\
\hline$(-2.25,5.00)$ & 10.00 & 10.00 & $1 \mathrm{E}-12$ & $(-1.21,-0.88)$ & $-1.24 \mathrm{E}-13$ & 0.00 & $1 \mathrm{E}-13$ \\
\hline$(0.25,5.00)$ & 9.99 & 10.00 & $1 \mathrm{E}-13$ & $(-0.46,-1.43)$ & $-1.33 \mathrm{E}-13$ & 0.00 & $1 \mathrm{E}-13$ \\
\hline$(2.75,5.00)$ & 10.00 & 10.00 & $1 \mathrm{E}-13$ & $(0.46,-1.43)$ & $-1.27 \mathrm{E}-13$ & 0.00 & $1 \mathrm{E}-13$ \\
\hline$(4.75,5.00)$ & 9.99 & 10.00 & $1 \mathrm{E}-13$ & $(1.21,-0.88)$ & $-5.98 \mathrm{E}-14$ & 0.00 & $1 \mathrm{E}-14$ \\
\hline
\end{tabular}

tinuity equation along the boundary, the unknown coefficients can be found. Hence, the solutions inside the domain can be retrieved by linear summation. In this example, 162 boundary nodes are used and $m$ is set as 40 . The velocity vector is demonstrated in Fig. 9. The tendency of flow field in Fig. 9 is almost the same as the results in [16]. In order to validate the results carefully, the velocity components along the boundary are computed and tabulated in Table 2. Since there is no analytical solution can be used, we computed the numerical solutions at another set of boundary nodes, which is totally different from the set of nodes for collocating the boundary conditions. From the table, we can find that the absolute errors are extremely small. Hence, it is proved that the proposed scheme, the combination of MCTM and the Laplacian decomposition, can obtained similar solutions as BEM and MFS based on Stokeslet [16].

\section{CONCLUSIONS}

In this paper, the combination of the Laplacian decomposition and the MCTM are proposed to solve the flow field governed by the Stokes equations.

At first, the Stokes equations are transformed to three Laplace equations by the Laplacian decomposition. Then the solutions of these Laplace equations are expressed by the linear combination of T-complete functions by following the idea of MCTM. Besides, the continuity equation is forced to be satisfied along the boundary to ensure the satisfaction of continuity equation inside the computational domain. The unknown coefficients of the solutions expressions are acquired by collocating the boundary condition and the continuity equation along the boundary. Once the coefficients are obtained, the flow field can be retrieved by linear summation.

The proposed numerical scheme is free from mesh and numerical quadrature. In addition, only boundary nodes are needed during the implementation, which can greatly reduce the computational cost. The numerical results of the four examples show that the proposed scheme can achieve high ac- curacy and be very stable. Besides, the distribution of pressure can be computed directly. Therefore, it is believed that the proposed scheme has great potential to be extended to realistic applications. It is well-known that performance of the MTCM is usually unsatisfactory when the problem with discontinuous boundary conditions is considered. Therefore, the solution expression in MCTM may cooperate with the enriched approach $[1,15]$ to increase the accuracy of solutions near the discontinuity. The combination of the MCTM and the enriched approach will be the future research topics.

\section{REFERENCES}

1. Alves, C. J. S. and Leitao, V. M. A., "Crack analysis using an enriched MFS domain decomposition technique," Engineering Analysis with Boundary Elements, Vol. 30, pp. 160-166 (2006).

2. Chen, W. and Fu, Z., "Boundary particle method for inverse Cauchy problems of inhomogeneous Helmholtz equations," Journal of Marine Science and Technology, Vol. 17, No. 3, pp. 157-163 (2009).

3. Chen, Y. W., Liu, C.-S., and Chang, J. R., "Applications of the modified Trefftz method for the Laplace equation," Engineering Analysis with Boundary Elements, Vol. 33, pp. 137-146 (2009).

4. Curteanu, A. E., Elliott, L., Ingham, D. B., and Lesnic, D., "Laplacian decomposition and the boundary element method for solving Stokes problems," Engineering Analysis with Boundary Elements, Vol. 31, pp. 501-513 (2007).

5. Fan, C. M. and Young, D. L., "Analysis of the 2D Stokes flows by the non-singular boundary integral equation method," International Mathematical Journal, Vol. 2, No. 12, pp. 1199-1215 (2002).

6. Fan, C. M., Young, D. L., and Chiu, C. L., "Method of fundamental solutions with external source for the eigenfrequencies of waveguides," Journal of Marine Science and Technology, Vol. 17, No 3, pp. 164-172 (2009).

7. Kevorkian, J., Partial Differential Equations: Analytical Solution Techniques, 2nd Ed., Springer-Verlag, New York (2000).

8. Kita, E. and Kamiya, N., "Trefftz method: an overview," Advances in Engineering Software, Vol. 24, pp. 3-12 (1995).

9. Li, Z. C., Lu, T. T., Huang, H. T., and Cheng, A. H. D., "Trefftz, collocation and other boundary methods-a comparison," Numerical Methods for Partial Differential Equations, Vol. 23, pp. 93-144 (2007).

10. Liu, C.-S., "A highly accurate collocation Trefftz method for solving the Laplace equation in the doubly connected domains," Numerical Methods for Partial Differential Equations, Vol. 24, pp. 179-192 (2008). 
11. Liu, C.-S., "A modified collocation Trefftz method for the inverse Cauchy problem of Laplace equation," Engineering Analysis with Boundary Elements, Vol. 32, pp. 778-785 (2008)

12. Liu, C.-S., "A modified Trefftz method for two-dimensional Laplace equation considering the domain's characteristic length," CMES: Computer Modeling in Engineering \& Sciences, Vol. 21, No. 1, pp. 53-65 (2007).

13. Liu, C.-S., "An effectively modified direct Trefftz method for 2D potential problems considering the domain's characteristic length," Engineering Analysis with Boundary Elements, Vol. 31, pp. 983-993 (2007).

14. Pozrikidis, C., Boundary Integral and Singularity Methods for Linearized Viscous Flow, Cambridge University Press, New York (1992).

15. Tsai, C. C. and Hsu, T. W., "A meshless numerical method for solving slow mixed convections in containers with discontinuous boundary data," International Journal for Numerical Methods in Fluids, Vol. 66, pp. $377-$ $402(2011)$

16. Young, D. L., Chen, C. W., Fan, C. M., Murugesan, K. and Tsai, C. C.,
"The method of fundamental solutions for Stokes flow in a rectangular cavity with cylinders," European Journal of Mechanics B/Fluids, Vol. 24, pp. 703-716 (2005).

17. Young, D. L., Chiu, C. L., Fan, C. M., Tsai, C. C., and Lin, Y. C., "Method of fundamental solutions for multidimensional Stokes equations by the dual-potential formulation," European Journal of Mechanics B/Fluids, Vol. 25, pp. 877-893 (2006).

18. Young, D. L., Jane, S. C., Lin, C. Y., Chiu, C. L., and Chen, K. C., "Solutions of 2D and 3D Stokes laws using multiquadrics method," Engineering Analysis with Boundary Elements, Vol. 28, pp. 1233-1243 (2007).

19. Young, D. L., Jane, S. J., Fan, C. M., Murugesan, K., and Tsai, C. C., "The method of fundamental solutions for 2D and 3D Stokes problems," Journal of Computational Physics, Vol. 211, pp. 1-8 (2006).

20. Zeb, A., Elliott, L., Ingham, D. B., and Lesnic, D., "The boundary element method for the solution of Stokes equations in two-dimensional domains," Engineering Analysis with Boundary Elements, Vol. 22, pp. 317-326 (1998). 